\title{
Resource allocation optimization of equipment development task based on MOPSO algorithm
}

\author{
ZHANG Xilin ${ }^{1,2}$, TAN Yuejin ${ }^{1}$, and YANG Zhiwei1,"* \\ 1. College of Systems Engineering, National University of Defense Technology, Changsha 410073, China; \\ 2. Business School, Jiangsu Normal University, Xuzhou 221116, China
}

\begin{abstract}
Resource allocation for an equipment development task is a complex process owing to the inherent characteristics, such as large amounts of input resources, numerous sub-tasks, complex network structures, and high degrees of uncertainty. This paper presents an investigation into the influence of resource allocation on the duration and cost of sub-tasks. Mathematical models are constructed for the relationships of the resource allocation quantity with the duration and cost of the sub-tasks. By considering the uncertainties, such as fluctuations in the sub-task duration and cost, rework iterations, and random overlaps, the tasks are simulated for various resource allocation schemes. The shortest duration and the minimum cost of the development task are first formulated as the objective function. Based on a multi-objective particle swarm optimization (MOPSO) algorithm, a multi-objective evolutionary algorithm is constructed to optimize the resource allocation scheme for the development task. Finally, an uninhabited aerial vehicle (UAV) is considered as an example of a development task to test the algorithm, and the optimization results of this method are compared with those based on non-dominated sorting genetic algorithm-II (NSGA-II), non-dominated sorting differential evolution (NSDE) and strength pareto evolutionary algorithm-II (SPEA-II). The proposed method is verified for its scientific approach and effectiveness. The case study shows that the optimization of the resource allocation can greatly aid in shortening the duration of the development task and reducing its cost effectively.
\end{abstract}

Keywords: resource allocation, equipment development task, multi-objective particle swarm optimization (MOPSO), development task simulation.

DOI: $10.21629 / J S E E .2019 .06 .09$

\section{Introduction}

In recent years, many countries have paid particular attention to the development of the manufacturing industry, as evidenced by the US "Industrial Internet", Germany's "Industry 4.0", and China's "Made in China 2025".

\footnotetext{
Manuscript received April 10, 2019.

*Corresponding author.

This work was supported by the National Natural Science Foundation of China (71690233).
}

The equipment manufacturing industry of a country is a representation of their manufacturing capabilities. Research and development (R\&D) is the core of equipment manufacturing. China attaches great importance to product development and has invested considerable resources for this purpose. In 2016, China invested 3.78 million R\&D personnel at a cost of 1567.67 billion yuan. When implementing equipment development tasks, it is necessary to allocate resources on a scientific basis ensuring their rational use and effectiveness for a smooth completion of the tasks, thereby promoting the equipment manufacturing industry.

The characteristics of an equipment development task include a huge investment of resources, numerous subtasks, complex network structures, and high degrees of uncertainty [1]. The resource allocation for an equipment development task refers to the rational allocation of limited high-end equipment development resources to each subtask in such a way as to ensure its smooth completion [2]. Many scholars have conducted in-depth research to optimize the resource allocation.

Due to a limited availability of resources, conflicts may arise during the execution of development tasks. At this time, resources should be allocated to sub-tasks according to a certain priority, and the priority of resources occupied by each sub-task should be considered [3,4]. In resourceconstrained multi-project scheduling, resource occupancy priority rules are related to factors such as network density, iteration intensity, and resource conflict level $[5,6]$. Wang et al. [7] studied the optimization of multi-priority rule combinations for multi-project scheduling problems under resource constraints. Chen et al. [8] studied the seven priority rule heuristics and justification techniques of a stochastic resource-constrained project scheduling problem (SRCPSP). It was proposed that the rationality of the SRCPSP depended on the difference between the priority rules and activity duration. Dixit et al. [9] solved the 
block spatial scheduling problem of shipbuilding projects using a priority rules-based simulation. Öner-Közen et al. [10] modeled the prioritization problem in a make to order production system and studied the impact of prioritization on the on-time probability and expected delay of orders. Chand et al. [11] proposed a genetic programmingbased hyper-heuristic algorithm for a resource-constrained project scheduling problem and compared the improved priority rules with the existing priority rules, demonstrating that the performance of the new method was significantly better than that using the existing priority rules.

The resource allocation problem is generally a multiobjective optimization problem, and the considered objectives include minimal cost, shortest construction period, highest efficiency, and minimal risk. Evolutionary algorithms include genetic algorithm (GA), particle swarm optimization (PSO), differential evolution (DE), fruit fly optimization algorithm (FOA), hybridizing grey wolf optimization (HGWO), and strength pareto evolutionary algorithm (SPEA), which can effectively solve problems such as optimization and prediction [12-15]. In the literature, multi-objective evolutionary algorithms are mainly used to optimize resource allocation problems [16]. Resources can be regarded as a set of capabilities required by a system or process, and may change dynamically with time and circumstance. On this basis, the specification and management methods of the subjects and resources required for the execution of a complex system were described [17]. Xiong et al. [18] established a multi-objective resource optimization and scheduling model for the scheduling problem of Chinese satellite systems and solved the model using an intelligent optimization algorithm. Lin et al. [19] used a hybrid algorithm of PSO and local search for the stochastic resource allocation. Yi et al. [20] constructed a mathematical model to solve the complex multi-objective resource allocation problems using an improved PSO algorithm. Beşikci et al. [21] studied the multi-project scheduling and resource combination problem under multi-model resource constraints that was optimized by a two-phase GA. Liu et al. [22] developed a column generation based distributed scheduling algorithm to solve the multi-mode resource constraint project scheduling problem. Kangaspunta et al. [23] developed an analytical model for resource consumption levels that can be used to identify the cost-effective subject combinations and tasks assigned to them. Guo et al. [24] considered use-cost, trading period, credibility, and resource consumption in the clustering model for manufacturing resources and used the improved cluster-based genetic algorithm for clustering. The manufacturing resource composition optimization of large complex equipment in group manufacturing was studied. Certa et al. [25] constructed a mathematical model of a human resource alloca- tion problem based on the project quality, human resource skills growth, and job team satisfaction. Then, the hierarchical sequence target planning method was used to solve the problem. Yaghoubi et al. [26] considered the resource allocation in a dynamic program evaluation and review technique (PERT) network with limited capabilities as a queuing theory problem and transformed the queuing network into a random network. The multi-objective programming model was constructed, with the least cost, shortest duration, and least amount of idling, as the objective function, and the resource allocation was optimized. The project network structure differs when different subsets of activities are selected. Tao et al. [27] studied the scheduling problem of multi-mode resource constraints by considering an alternative project structure, and developed a mixedelement heuristic algorithm, which effectively solved the problem.

There are uncertainties in the allocation of resources for an equipment development task. For example, it is possible that construction period, cost, and rework probability are uncertain. It is necessary to consider the uncertainties in the study of an allocation method. In the current literature, the problem of resource allocation is considered under uncertain conditions in the manufacturing process. Laslo et al. [28] studied the resource allocation of complex projects in uncertain environments based on the system dynamics method. Wang et al. [29] analyzed the performance of priority rules under the condition of random activity duration and studied the relationship between the project characteristics and priority rules. Leus et al. [30] proposed a resource allocation model under the uncertainty of the construction period and applied the branchand-bound algorithm to solve the model. Usually, the activity duration depends on the amount of resources allocated, that is, the larger the amount of allocated resources is, the shorter the working time is. Liu et al. [31] modeled a project scheduling problem of job time-dependent resource allocation based on a linear relationship between the job time and resource allocation. During the execution of the project, there will be some uncertainties in the duration of each activity. Chakrabortty et al. [32] used random variables with different probability distribution functions to represent the activity duration, and studied the resource constraint project scheduling problem with an uncertain activity duration. Bruni et al. [33] assumed that the activity duration is affected by the interval uncertainty, and proposed a general decomposition method to solve the robust correspondence problem of resource-constrained project scheduling problems.

The current literature mainly includes studies on the resource allocation in the manufacturing processes, and there are only relatively few studies relating to resource allo- 
cation for the development tasks. In terms of the quantity of resource allocations to achieve the desired subtask duration and cost, most of the literature focuses on manufacturing-related methods, whereby it is assumed that the sub-task duration and cost are linearly related to the quantity of resource allocation. However, a development task differs from a manufacturing task because it essentially involves innovation. Accordingly, in a development task, the sub-task duration and cost are not linearly related to the quantity of the resource allocation. In terms of research methods, the current literature mainly constructs a task resource allocation model through mathematical modeling. On this basis, an analytical model or a heuristic method is used to solve a mathematical model. However, an equipment development task has many influencing factors, a strong randomness, and a complex task network. Therefore, there is a great uncertainty in the development task process as a whole. The constructed mathematical models are not capable of reflecting the real operation of a development task under different resource allocation schemes.

This study investigates the impact of resource allocation on the duration and cost of a development task and constructs mathematical models for the relationships of the resource allocation quantity with the duration and sub-task cost. The resource allocation of an equipment development task is evaluated by its duration and cost (simulation outputs) and is optimized based on the multi-objective particle swarm optimization (MOPSO) algorithm.

This paper is organized as follows. In Section 2, the problem is described in detail, mathematical models of the relationships of the resource allocation quantity with the sub-task duration and cost are constructed, and operation effects of the development tasks under different resource allocation schemes are evaluated by simulation, based on the design structure matrix (DSM). In Section 3, the development task simulation model is embedded into the MOPSO algorithm. The average duration and average cost (simulation outputs) are used as the fitness evaluation indices, and the resource allocation is optimized using the MOPSO algorithm. In Section 4, the algorithm is applied in the resource allocation of an uninhabited aerial vehicle (UAV) development task, and the scientific approach and effectiveness of the methods are verified. Section 5 presents a brief summary of the findings of the study and the prospects for future research.

\section{Description and analysis of development task resource allocation problem}

\subsection{Description of problem}

Renewable resources are studied in this paper. The human and equipment resources for renewables are limited at any given point of time and are not consumed as the task progresses. The purpose of this study is to determine the various uncertainties of a development task under the conditions of a total amount of resources, and how to allocate resources to each sub-task. The problem can be specifically described as follows: an equipment development task includes multiple sub-tasks, and there are various uncertain relationships among the sub-tasks: serial, parallel, rework iteration, and overlap execution. The total amount of resources available for the entire development task is limited. The quantity of resources allocated to a sub-task affects its duration and cost. The higher the resource allocation to a sub-task is, the shorter the duration of the sub-task and the higher the cost will be. When the quantity of resources allocated to a sub-task is constant, the duration and cost of the sub-task fluctuate randomly within a certain range. Under the given development task, the resource allocation of the equipment development task is optimized for the shortest average duration and lowest average cost. The quantity of the resources allocated to each sub-task is then obtained.

\subsection{Relationship between sub-task duration and cost, and resource allocation quantity}

\subsubsection{Relationship between sub-task duration and resource allocation quantity}

For the resource allocation of a development task, most of the current literature assumes that the duration of the subtasks is constant or obeys a certain distribution. Usually, during the execution of the equipment development task, the quantity of resources allocated to a sub-task affects its duration, and a mathematical model of the relationship between the sub-task duration and quantity of resource allocation can be constructed.

In general, the higher the amount of resources allocated to a sub-task is, the shorter the sub-task duration will be. However, as the quantity of resources increases, the reduction rate of the duration becomes progressively slower, and it requires teamwork to complete the execution of a subtask. Therefore, the quantity of resources allocated to a sub-task needs to reach a certain scale, termed the minimum quantity of resource demand, so that the sub-task can be executed. With an increased quantity of resources allocated to a sub-task, the execution time decreases. However, while executing the sub-task, the team members have requirements such as knowledge accumulation and information transmission. The efficiency of resources allocated to the sub-tasks decrease with the increased quantity of resources. However, due to factors such as the preparation, internal activity sequence, and information transmission, the shortening of the sub-task duration does not continue 
indefinitely, and usually there is a finitely short sub-task duration. Once the shortest duration is reached, additional resources for a sub-task do not shorten the duration, and the corresponding resource amount is termed the maximum quantity of resource demand (MQRD) of the sub-task. The relationship between the sub-task duration and quantity of resource allocation is shown in Fig. 1.

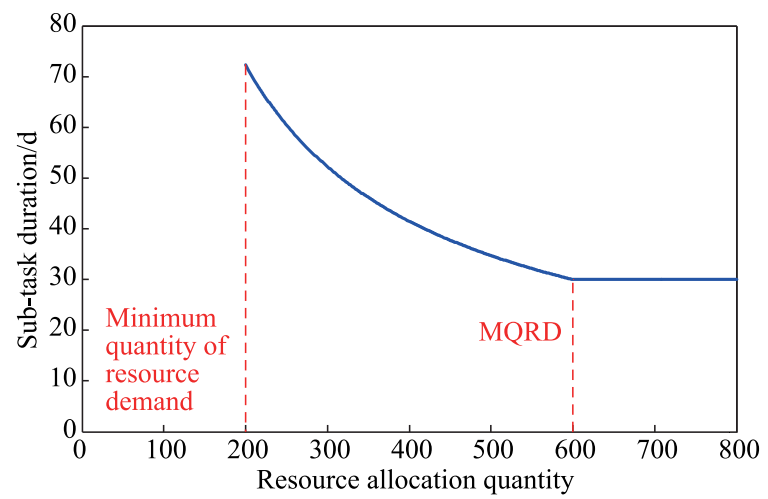

Fig. 1 Relationship between the sub-task duration and quantity of resource allocation

The total amount of workload required to complete a sub-task is independent of the quantity of resources allocated to it. The mathematical model for the relationship between the duration of sub-task $i$ and resource allocation quantity can be expressed as follows:

$$
D_{i}=\left\{\begin{array}{l}
\infty, \quad R_{i}<R L_{i} \\
T M_{i} \cdot\left(\frac{R U_{i}}{R_{i}}\right)^{\alpha}, \quad R L_{i} \leqslant R_{i} \leqslant R U_{i} \\
T M_{i}, \quad R_{i}>R U_{i}
\end{array}\right.
$$

where $D_{i}, R_{i}$, and $T M_{i}$ are the duration, resource allocation quantity, and minimum duration, respectively, of the sub-task $i$; $R L_{i}$ is the minimum quantity of the resource demand when the sub-task $i$ can be executed, that is, the minimum quantity of the resource demand of the sub-task $i$; $R U_{i}$ is the number of resources allocated to the sub-task $i$ when the duration of the sub-task $i$ is the shortest, that is, the MQRD of the sub-task $i$; and $\alpha$ is the adjustment factor in the range $[0,1]$. The larger the value of $\alpha$ is, the greater the marginal utility when adding a unit resource to the sub-task will be, and the sub-task duration is shortened more significantly.

\subsubsection{Relationship between sub-task cost and resource allocation quantity}

During the execution of the equipment development task, both the quantity of resources allocated to a sub-task and efficiency of the unit resources differ. In general, the marginal utility of a resource decreases as the quantity of resources allocated increases. Therefore, the cost of completing a sub-task varies with the quantity of resources assigned to it.

It is assumed that the cost of a sub-task occupying a unit resource per unit time is a fixed value, and the cost required to complete a sub-task can be calculated using the unit cost, resource quantity, and occupation time of the occupied resource. If an additional resource is allocated to the sub-task after it reaches the shortest duration, the cost of completing the sub-task increases linearly. The relationship between the sub-task cost and quantity of resource allocation is shown in Fig. 2.

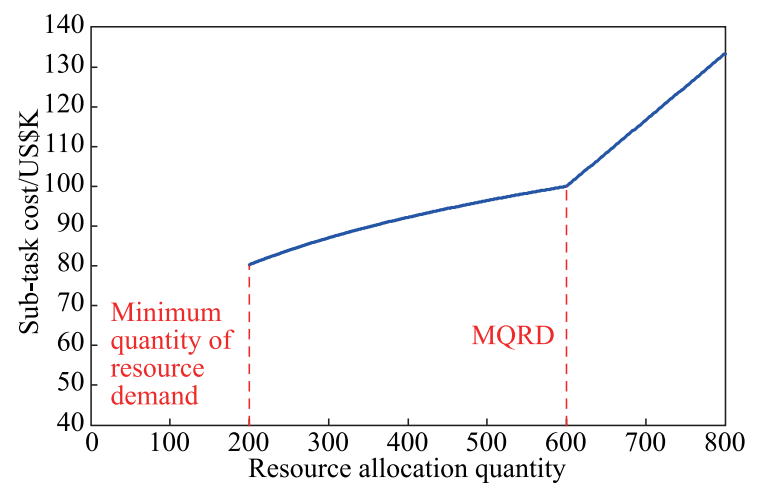

Fig. 2 Relationship between the sub-task cost and quantity of resource allocation

The cost per unit time of the unit resource occupied by the sub-task is calculated by the cost of the sub-task when the quantity of resource demand is a maximum. The cost of the sub-task $i$ occupying the unit resource per unit time can be expressed as

$$
C P_{i}=\frac{C M_{i}}{R U_{i} \cdot T M_{i}}
$$

where $C P_{i}$ is the cost of the sub-task $i$ occupying the unit resource per unit time and $C M_{i}$ is the cost corresponding to the MQRD of the sub-task $i$.

The cost of the sub-task is proportional to the quantity of occupied resources and occupation time. If $R L_{i} \leqslant R_{i} \leqslant$ $R U_{i}$, the cost of completing the sub-task $i$ can be expressed as

$$
C_{i}=C P_{i} \cdot R_{i} \cdot D_{i}=C M_{i} \cdot\left(\frac{R_{i}}{R U_{i}}\right)^{1-\alpha}
$$

where $C_{i}$ is the cost of completing the sub-task $i$.

If $R_{i}>R U_{i}$, although the quantity of resources allocated to the sub-task $i$ increases, the duration is no longer shortened. At this time, the cost of completing the sub-task $i$ can be expressed as

$$
C_{i}=C P_{i} \cdot R_{i} \cdot D_{i}=\frac{C M_{i} \cdot R_{i}}{R U_{i}} .
$$

Therefore, the relationship between the cost of completing the sub-task $i$ and the quantity of resource allocation 
can be expressed as

$$
C_{i}=\left\{\begin{array}{l}
\infty, \quad R_{i}<R L_{i} \\
C M_{i} \cdot\left(\frac{R_{i}}{R U_{i}}\right)^{1-\alpha}, \quad R L_{i} \leqslant R_{i} \leqslant R U_{i} \\
\frac{C M_{i} \cdot R_{i}}{R U_{i}}, \quad R_{i}>R U_{i}
\end{array}\right.
$$

\subsection{Evaluation of development task operation performance under different resource allocation schemes}

When different resources are allocated to sub-tasks of an equipment development task, the efficiency of resources is different, and the operation effect of the development task is also different. While optimizing the resource allocation, we need to evaluate the operational effectiveness of different resource allocation schemes. In this study, the duration and cost of the development task are selected as the evaluation indicators of the operation effect of the task. For the algorithm of this paper, the objective function is based on the minimum average duration and minimum average cost of the simulation output of the development task:

$$
\min (E(T D), E(T C))
$$

where $E(T D)$ and $E(T C)$ are the average duration and cost, respectively, of the simulation output of the development task.

Because of the complexity of the development task and many uncertain factors, it is difficult to solve and analyze the duration and cost with a mathematical model. In this study, mathematical models for the relationships of the resource allocation quantity with the sub-task duration and cost are first constructed. Then, using these as the inputs for the sub-task duration and cost of the simulation model, various relationships between the sub-tasks, rework iteration, overlapping execution, resource constraints, learning effects, and resource occupancy priority, are considered. A simulation method from [34] is used to obtain the duration and cost of the development task under different resource allocation schemes.

\section{Optimization of resource allocation for development task based on MOPSO algorithm}

The MOPSO algorithm is an evolutionary technology based on swarm intelligence, which effectively solves the problems of slow convergence and is easy to fall into local optimum in high-dimensional and multi-objective evolution. The algorithm has a strong versatility and memory ability, and makes full use of the individual's local optimal information and the group's overall optimal information collaborative search. At present, MOPSO has been applied to many optimization areas, especially in the field of engineering optimization. The development task resource allocation optimization problem studied in this paper is a highdimensional, multi-objective optimization problem, which is very suitable for being solved with the MOPSO algorithm.

\subsection{Algorithm framework}

MOPSO is a heuristic algorithm based on information sharing among the particles [35-37]. Each particle represents a potential solution to the multi-objective optimization problem. In the optimization problem of resource allocation for a development task, the particle characteristics are represented by three indices, such as the position, velocity, and fitness. The non-inferior solution set is filtered from the initial particle swarm. A particle that is not dominated by other particles is placed into the non-inferior solution set. The particle updates its position by tracking the individual optimal particle $p$ Best (the position where the calculated fitness value in the position experienced by the individual is optimal) and the group optimal particle $g$ Best (the randomly selected particle position in the group of the non-inferior solution set). The individual optimal particle $p$ Best and current non-inferior solution sets are merged, and the new non-inferior solution sets are screened out through continuous iteration until a satisfactory solution is obtained.

In this study, the resource allocation scheme is evaluated by the average duration and cost of the task (simulation outputs), and the MOPSO algorithm is used to optimize the resource allocation scheme for the equipment development task. The particles are coded according to the resource allocation scheme. The particles are mutated with a decreasing probability, and a satisfactory resource allocation scheme is sought through continuous iteration. The pseudo code of the algorithm is shown as follows.

Initialize

Randomly generate each particle's velocity and position

Evaluate the fitness value of each particle

Fill the $p$ Best of each particle with its current position

for $i t=1$ : MaxIt

for $k=1: n \mathrm{Pop}$

Select $g$ Best

Update particle velocity $v_{k}$

Update particle position $x_{k}$

if rand $<p m$

Apply mutation

end if

Evaluation of particle $k$

Update $p$ Best 


\section{end for}

Add non-dominated particles to repository Determine domination of new repository members Keep only non-dominated members in the repository Update grid and grid indices

$$
\text { if } \text { numel }(\text { rep })>n R e p
$$

Delete $($ numel $(r e p)-n R e p)$ repository members end if end for

\subsection{Algorithm implementation}

\subsubsection{Particle coding}

Each particle position represents a resource allocation scheme for an equipment development task, and each dimension of a particle position represents the quantity of resources allocated to a sub-task. The velocity of each particle represents the change in the resource allocation quantity of the corresponding resource allocation scheme, and the dimension of the particle velocity represents the change in speed of the quantity of resources allocated to the subtask. For the optimization of the resource allocation, the expressions of the position and velocity of the particles are

$$
\begin{aligned}
x_{k} & =\left(x_{k 1}, x_{k 2}, \ldots, x_{k j}, \ldots, x_{k n}\right) \\
v_{k} & =\left(v_{k 1}, v_{k 2}, \ldots, v_{k j}, \ldots, v_{k n}\right)
\end{aligned}
$$

where $k$ is the particle number corresponding to the resource allocation scheme number; $x_{k}$ is the position of the particle $k$ corresponding to the resource allocation scheme $k ; j$ is the sub-task number; $x_{k j}$ is the resource allocation for the sub-task $j$ in the particle $k ; v_{k}$ is the velocity of the particle $k$ corresponding to the change in speed of the resource allocation scheme $k ; v_{k j}$ is the change in speed of the resource allocation for the sub-task $j$ in the particle $k$.

\subsubsection{Fitness evaluation}

In this study, the performance of the development task under different resource allocation schemes is evaluated by indicators, such as the average duration and average cost. Because it is difficult to carry out the mathematical modeling directly to solve the duration and cost of the development task, this study employs DSM to simulate the development task using the resource allocation scheme of each particle. The simulation modeling is described in [34]. The average duration and average cost of the simulation output are used as the fitness evaluation indices of each particle, and the resource allocation scheme of the development task corresponding to each particle is non-dominated.

\subsubsection{Update and correction of particle velocity and position}

As the quantity of resources allocated to a sub-task is required to be an integer, it is necessary to use an integer for the value of each dimension of the particle velocity. To prevent particles from flying too fast from one area of the search space to another, the velocity of each dimension of a particle (the change in speed of the resource allocation quantity) is limited to the range $\left[v \min _{j}, v \max _{j}\right]$. Here, the velocity can be a positive or negative value, and therefore $v \min _{j}<0$ and $v \max _{j}>0$. If the velocity of the dimension $j$ of the particle $k$ is less than $v \min _{j}, v_{k j}(t)$ equals $v \min _{j}$; and if the velocity of the dimension $j$ of the particle $k$ is larger than $v \min _{j}, v_{k j}(t)$ equals $v \max _{j}$. The updated formula for the velocity of particle $k$ is

$$
\begin{array}{r}
v \text { New }=\operatorname{round}\left[\omega \cdot v_{k}(t)+c_{1} \cdot r_{1} \cdot\left(p \operatorname{Best}_{k}(t)-x_{k}(t)\right)+\right. \\
\left.c_{2} \cdot r_{2} \cdot\left(p \operatorname{Best}_{k}(t)-x_{k}(t)\right)\right] \\
v_{k j}(t+1)= \begin{cases}v \min _{j}, & v \operatorname{New}_{j} \leqslant v \min _{j} \\
v \max _{j}, & v \operatorname{New}_{j} \geqslant v \max _{j} \\
v \operatorname{New}_{j}, & v \min _{j}<v \operatorname{New}_{j}<v \max _{j}\end{cases}
\end{array}
$$

where $v_{k j}(k+1)$ is the velocity of the dimension $j$ of the particle $k$ in the $(t+1)$ th iteration; $v_{k}(t)$ is the velocity of the particle $k$ in the $t$ th iteration; $x_{k}(t)$ is the position of the particle $k$ in the $t$ th iteration; $\omega$ is the inertia weight; $c_{1}$ and $c_{2}$ are the personal and global learning coefficients, respectively; $p \operatorname{Best}_{k}(t)$ is the personal best position in the $t$ th iteration; and $g \operatorname{Best}_{k}(t)$ is the swarm best position in the $t$ th iteration.

The inertia weight indicates how much the original velocity of the particle can be retained. In the iterative process, the inertia weight value " $\omega$ " generally decreases as the number of iterations increases in order to ensure a strong global search capability at the beginning of the algorithm and a strong local search capability at the end of the algorithm. In this paper, $\omega$ is linearly reduced with the number of iterations, and its formula can be expressed as

$$
\omega_{t+1}=\omega \operatorname{damp} \cdot \omega_{t}
$$

where $\omega_{t+1}$ and $\omega_{t}$ are the inertia weights in the $(t+1)$ th iteration and the $t$ th iteration; $\omega$ damp is the inertia weight damping rate.

For the particle position calculation, since the quantity of resources allocated to each sub-task is limited, each dimension position (quantity of resource allocation) of the particle is limited to the range $\left[x \min _{j}, x \max _{j}\right]$. If the position of the dimension $j$ of the particle $k$ is less than $x \min _{j}, x_{k j}(t)$ equals $x \min _{j}$; if the position of the dimension $j$ of the particle $k$ is greater than $x \max _{j}, x_{k j}(t)$ equals $x \max _{j}$. The updated formula for the position of particle $k$ is

$$
x \mathrm{New}=x_{k}(t)+v_{k}(t+1)
$$




$$
x_{k j}(t+1)= \begin{cases}x \min _{j}, & x \mathrm{New}_{j} \leqslant x \min _{j} \\ x \max _{j}, & x \mathrm{New}_{j} \geqslant x \max _{j} \\ x \mathrm{New}_{j}, & x \min _{j}<x \mathrm{New}_{j}<x \max _{j}\end{cases}
$$

$$
l b_{r}=\max \left\{\left(x_{r}-\operatorname{round}\left(p m \cdot\left(x \max _{r}-x \min _{r}\right)\right)\right), x \min _{r}\right\}
$$

where $x_{k j}(t+1)$ is the position of the dimension $j$ of the particle $k$ in the $(t+1)$ th iteration.

\subsubsection{Mutation operation}

A MOPSO algorithm can quickly yield optimization results due to its rapid convergence. However, it may also cause the particle population to fall into a local optimal solution without the possibility of jumping out. To overcome this defect, a mutation operation is performed on the particles in the iterative process of the algorithm. At the initial iteration stage, there is a large gap between the solution and optimal solution sets, requiring a large-scale search. At this point, to expand the search scope of the resource allocation scheme, a higher particle mutation probability is used. As the number of iterations increases, the gap between the obtained and optimal solution sets is gradually reduced. To make the algorithm converge quickly, the mutation probability of the particles is gradually reduced. Therefore, as the number of iterations increases, the mutation probability of the particles decreases. The mutation probability of the particles can be expressed as

$$
p m(t)=\left(1-\frac{t-1}{\text { MaxIt }-1}\right)^{\frac{1}{\mu}}
$$

where $p m(t)$ is the mutation probability in the $i$ th iteration; MaxIt is the maximum iteration number of the MOPSO algorithm; $\mu$ is the adjustment factor.

During the particle mutation process, the position $r$ of the particle is selected randomly, and the variation range of the corresponding resource allocation quantity is as follows:

$$
u b_{r}=\min \left\{\left(x_{r}+\operatorname{round}\left(p m \cdot\left(x \max _{r}-x \min _{r}\right)\right)\right), x \max _{r}\right\}
$$

where $l b_{r}$ and $u b_{r}$ are the lower and upper limits of the variation range of the quantity of resource allocation corresponding to the particle position $r$, respectively; $x_{r}$ is the current quantity of resource allocation corresponding to the particle position $r ; x \max _{r}$ and $x \min _{r}$ are the maximum and minimum values of the quantity of resource allocation corresponding to the particle position $r$, respectively.

The quantity of resource allocation corresponding to the particle position $r$ is assumed to be a random integer between $l b_{r}$ and $u b_{r}$, and a new resource allocation scheme for the development task is generated. At this point, the mutation process of the particles is completed.

\section{Case study}

\subsection{Case description}

A case study is performed in this section on the resource allocation problem of a UAV development task. The UAV development task includes 14 sub-tasks, and the data (e.g., duration, cost) and resource demands of each sub-task are shown in Table 1 [38]. The MQRDs of the sub-tasks are also provided. If the quantity of resources allocated to a sub-task is greater than its MQRD, its duration can no longer be shortened. The minimum quantity of resource demand is $60 \%$ of the MQRD. If the amount of resources

\begin{tabular}{|c|c|c|c|c|c|c|c|c|}
\hline \multirow{2}{*}{ ID } & \multirow{2}{*}{ Sub-task name } & \multicolumn{3}{|c|}{ Minimum duration/d } & \multicolumn{3}{|c|}{ Maximum cost/US\$K } & \multirow{2}{*}{ MQRD } \\
\hline & & $D M_{o}$ & $D M_{m}$ & $D M_{p}$ & $C M_{o}$ & $C M_{m}$ & $C M_{p}$ & \\
\hline 1 & Prepare UAV preliminary design requirements and objectives & 1.9 & 2 & 3 & 8.6 & 9 & 13.5 & 400 \\
\hline 2 & Create UAV preliminary design configuration & 4.75 & 5 & 8.75 & 5.3 & 5.63 & 9.84 & 600 \\
\hline 3 & Prepare surfaced models \& internal drawings & 2.66 & 2.8 & 4.2 & 3 & 3.15 & 4.73 & 600 \\
\hline 4 & Perform aerodynamics analyses \& evaluation & 9 & 10 & 12.5 & 6.8 & 7.5 & 9.38 & 400 \\
\hline 5 & Create initial structural geometry & 14.3 & 15 & 26.3 & 128 & 135 & 236 & 600 \\
\hline 7 & Develop freebody diagrams \& applied loads & 7.2 & 8 & 10 & 11 & 12 & 15 & 500 \\
\hline 8 & Perform weights \& inertia analysis & 4.75 & 5 & 8.75 & 8.9 & 9.38 & 16.4 & 300 \\
\hline 9 & Perform stability and control analyses \& evaluation & 18 & 20 & 22 & 20 & 22.5 & 24.8 & 500 \\
\hline 10 & Develop freebody diagram \& applied loads & 9.5 & 10 & 17.5 & 21 & 22.5 & 39.4 & 400 \\
\hline 11 & Establish internal load distributions & 14.3 & 15 & 26.3 & 21 & 22.5 & 39.4 & 500 \\
\hline 12 & Evaluate structural strength, stiffness \& life & 13.5 & 15 & 18.8 & 41 & 45 & 56.3 & 400 \\
\hline 13 & Preliminary manufacturing planning $\&$ analyses & 30 & 32.5 & 36 & 214 & 232 & 257 & 600 \\
\hline
\end{tabular}
allocated to a sub-task is less than its minimum quantity of resource demand, the sub-task cannot be executed.

Table 1 Duration, cost, and resource requirements of a UAV development task 
The minimum duration is the corresponding duration when the resource allocated to the sub-task is MQRD. Assuming that the distribution function of the minimum duration is triangular, the optimistic duration, the most probable duration, and the pessimistic duration of the minimum duration are respectively represented by $D M_{o}, D M_{m}$ and $D M_{p}$. The maximum cost is the corresponding cost when the resource allocated to the sub-task is MQRD. Assuming that the distribution function of the maximum cost is triangular, the optimistic cost, the most probable cost, and the pessimistic cost of the maximum cost are respectively represented by $C M_{o}, C M_{m}$ and $C M_{p}$.

For the resource allocation of the UAV development task, both the quantity of resources allocated to a sub-task and the parameters, such as the duration and cost of the sub-task, differ. Assuming that different resource quantities are allocated to the sub-task, the function distributions of the corresponding duration and the cost of the sub-task are triangular. At this point, the parameters, such as the optimistic duration, most likely duration, pessimistic duration, optimistic cost, most likely cost, and pessimistic cost are calculated using the method described in Section 2.2. The DSM, rework probability matrix, rework influence matrix, overlap rate matrix, overlap influence matrix, and learning curve of the UAV development task are provided in [34] and [39].

\subsection{Optimization of the resource allocation for the development task}

\subsubsection{Parameter setting}

The total quantity of available resources of the equipment development task is 1000 , and the development task pro-

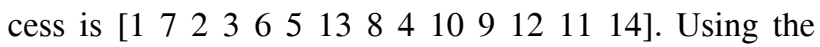
shortest duration and lowest cost of the development task as the objective function, the development task resource allocation scheme of the UAV is optimized based on the MOPSO algorithm. The literature shows that when the initial inertia weight $\omega$ is set to 0.729844 , the personal learning coefficient $c_{1}$ and the global learning coefficient $c_{2}$ are set to 1.49618 , the MOPSO algorithm has the best performance $[40,41]$. Since the traditional MOPSO algorithm is improved in this paper, the optimization results are not optimal under the parameters combination. The comparison of several experiments shows that under the conditions given in the example of this paper, $\omega$ is set to $0.8, c_{1}$ and $c_{2}$ are set to 1.5 , and the optimization results are the optimal. The remaining parameters of the MOPSO algorithm are as follows: 200 iterations; population size of 100; repository size of 50; inertia weight damping rate of 0.99 ; initial mutation rate adjustment factor $\mu$ of 0.1 that decreases as the number of iterations increases.

The method adopted in this study must evaluate the resource allocation scheme continuously during the simulation. The higher the number of simulations for each scenario is, the more stable the outputs of the simulation are. The running time of the algorithm is predominantly the simulation time. The higher the number of simulations of each scheme is, the longer the running time of the algorithm will be. To this end, the Pareto optimal solution set is obtained by running the MOPSO algorithm, whereby the number of simulations of the development task under various resource allocation schemes is set to 200 . Then, each optimal solution in the Pareto set is mapped to the corresponding resource allocation scheme, and the number of simulations is set to 5000 , and a fine simulation is performed on each scheme. The Pareto optimal solution set is recalculated according to the duration and cost of the fine simulation of the development task. The convex set is calculated, and the intersection of the Pareto optimal solution and convex sets is selected as the best solution set.

\subsubsection{Optimization results and analysis}

The maximum resource demand of each sub-task is used as the pre-optimization scheme for comparison with the optimized scheme. Non-dominated sorting genetic algorithm-II (NSGA-II), non-dominated sorting differential evolution (NSDE) and strength pareto evolutionary algorithm-II (SPEA-II) are commonly used algorithms in multi-objective optimization. To verify the optimization effect of the proposed method, the optimized scheme of this paper is compared with both the scheme before optimization and the scheme optimized by NSGA-II, NSDE and SPEA-II. Before the optimization, the development task duration and cost, $(D, C)$, are $(118.1,690.1)$; those obtained by the proposed optimization method are (135.1, 622.7), (121.2, 626.9), (118.4, 627.9), (111.8, 650.3), and (113.2, 644.5); those obtained by the NSGA-II optimization are $(132.2,628.9),(112.7,642.2),(123.3,630.5)$, (118.4, 633.9), (114.9, 637.6), and (121.3, 631.0); those obtained by the NSDE optimization are (120.3, 633.8), (115.1, 641.3), (124.2, 631.2), (122.1, 632.3), (117.3, 636.4), and (111.5, 649.4); and those obtained by the SPEA-II optimization are (133.1, 629.9), (124.8, 630.3), (123.9, 630.6), (115.5, 640.7), (122.0, 632.8), and (112.0, 647.8). A comparison of the duration and cost of the UAV development task obtained by optimizing the resource configuration by different algorithms is shown in Fig. 3. It is evident that the optimization of the resource allocation by the above method can shorten the duration and reduce the cost of the development task significantly. Moreover, the cost of the development task optimized by the MOPSO al- 
gorithm is less than that obtained by the NSGA-II, NSDE and SPEA-II optimization, indicating that the optimization effect based on the MOPSO algorithm is the best.

The resource allocation schemes of the UAV development task before and after the optimization, and corresponding parameters, such as duration, cost, resource conflict times, and rework times, are provided in Table 2. The degree of resource conflicts can be reduced by optimizing the resource allocation of the development task. Compared with the pre-optimization scheme, the average number of resource conflicts of the scheme optimized by the MOPSO algorithm is greatly reduced by $64.9 \%, 63.2 \%$, $59.8 \%, 38.1 \%$, and $43.3 \%$. However, there is no obvious change in the trend of the average rework times of the optimized development task.

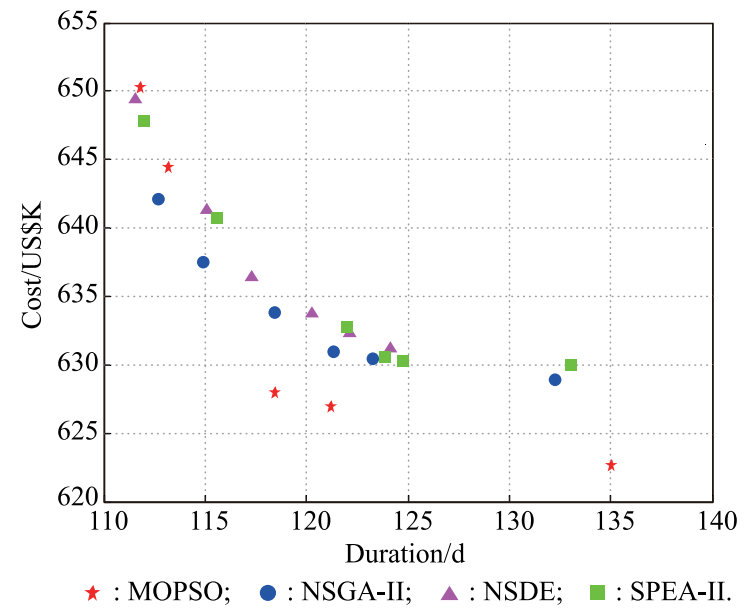

Fig. 3 Comparison of the duration and cost obtained by different optimization algorithms

Table 2 Comparison of operation effects before and after optimization

\begin{tabular}{ccccc}
\hline Resource allocation scheme & Duration/d & Cost/US\$K & $\begin{array}{c}\text { Average occurrence } \\
\text { of resource conflicts }\end{array}$ & $\begin{array}{c}\text { Average number } \\
\text { of reworks }\end{array}$ \\
\hline 400600600400600500500300500400500400600500 & 118.1 & 690.1 & 29.1 & 11.2 \\
240361365240372341300182301243308400371300 & 135.1 & 622.7 & 10.1 & 10.7 \\
353360368242360306304180300245329400366427 & 121.2 & 626.9 & 10.7 & 10.6 \\
354363360297368316302181300244330400367500 & 118.4 & 627.9 & 1.7 & 10.9 \\
400369377313432318302190308241353240402500 & 111.8 & 650.3 & 16.5 & 13.5 \\
369364385290437308301180312240374241365494 & 113.2 & 644.5 & 16.5 & 13.4 \\
\hline
\end{tabular}

When the total quantity of resources is limited, there may be resource conflicts during the execution of the development task. A few sub-tasks that are restricted by resources cannot be executed in time, and therefore for these sub-tasks, it is necessary to wait until other sub-tasks are completed so that resources can be released for their execution. In order to more intuitively represent the development task execution, the Gantt chart under various schemes is drawn. The Gantt charts of the development task execution under the pre-optimization and optimized resource allocation schemes are shown in Fig. 4.

When the amount of resources allocated to each sub-task is the maximum of its demand, the resource conflict is the most significant. At this point, some sub-tasks cannot be executed in parallel due to resource conflicts, resulting in a reduction in the number of sub-tasks executed in parallel.

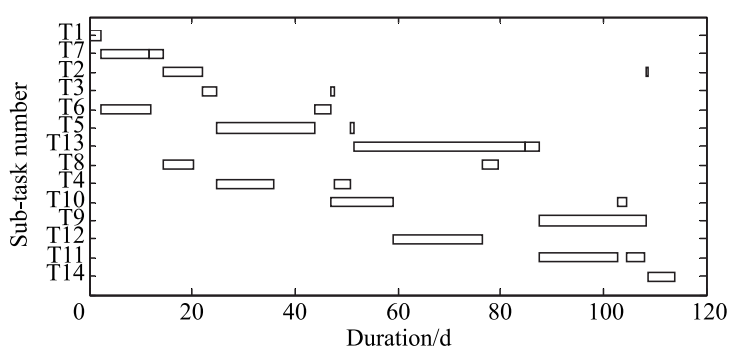

(a) Gantt chart before resource allocation optimization

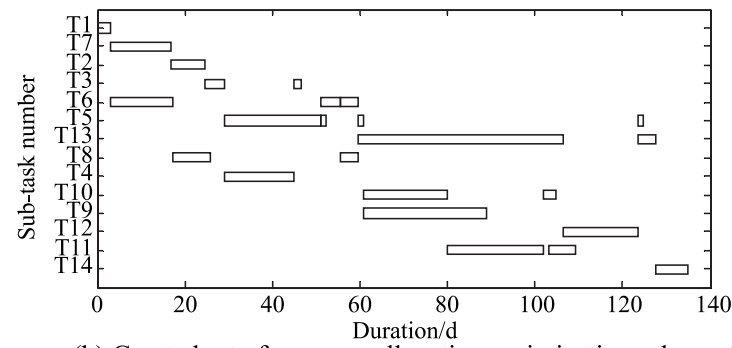

(b) Gantt chart of resource allocation optimization scheme 1

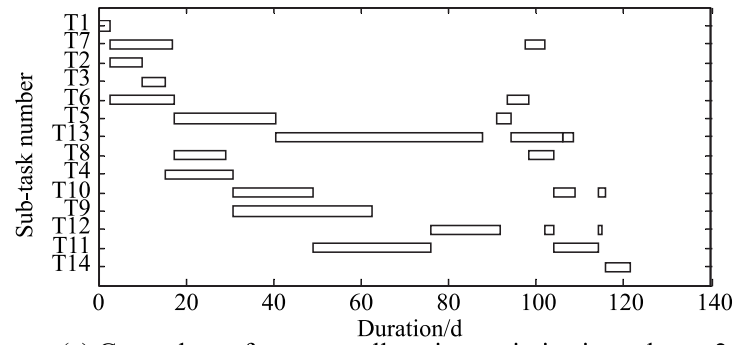

(c) Gantt chart of resource allocation optimization scheme 2

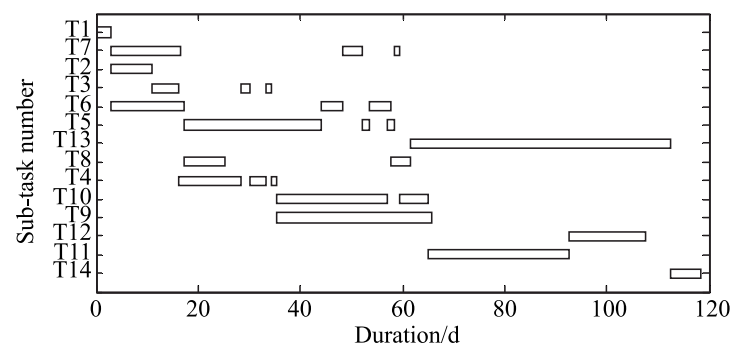

(d) Gantt chart of resource allocation optimization scheme 3 


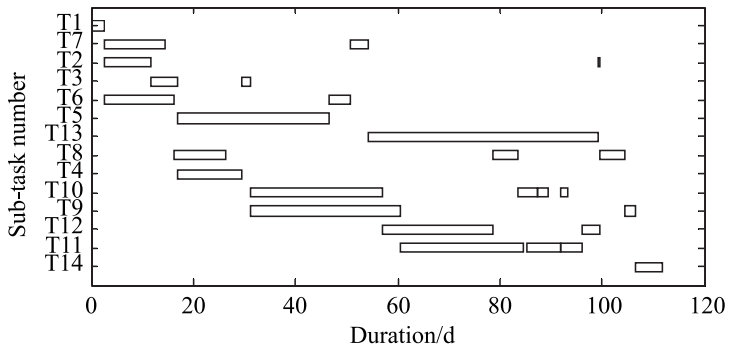

(e) Gantt chart of resource allocation optimization scheme 4

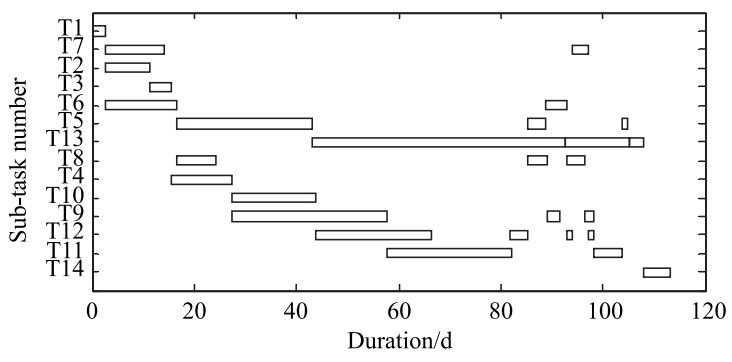

(f) Gantt chart of resource allocation optimization scheme 5

Fig. 4 Gantt charts of development task execution before and after optimization

Although the duration of each sub-task is minimal, the duration of the entire development task is not minimal. Moreover, in this case, the cost demand to complete each sub-task is relatively large, and consequently, the cost required for the completion of the entire development task is also relatively large. Therefore, the scheme is usually not a satisfactory solution.

By optimizing the resource allocation of the development task, the cost of some sub-tasks can be reduced, and consequently, the cost of the whole development task can also be effectively reduced. Therefore, it is critical to allocate resources reasonably to sub-tasks according to the execution process of the development tasks. Reducing the quantity of resources leads to an increased duration of the sub-tasks. However, this simultaneously reduces the resource conflicts among the sub-tasks, thereby increasing the number of sub-tasks executed in parallel, which can aid in the reduction of the duration of the development task. However, if low cost is targeted, it is necessary to restrict the quantity of resources allocated to the sub-tasks, which in turn increases the duration of the development task. The above case study on the UAV development task demonstrates that the optimization of the resource allocation can shorten the duration, and reduce the cost and degree of resource conflicts among the sub-tasks effectively.

\section{Conclusions}

This paper provides an analysis of the impact of the quantity of resource allocation on the duration and cost of a sub-task, and mathematical models for the relationships between the quantity of resource allocation and duration and cost of a sub-task. The models consider the marginal utility of resources, as well as the maximum and minimum numbers of the resource demands for sub-tasks. Based on the traditional simulation model for a development task, the influence of the quantity of resource allocation on parameters, such as the sub-task schedule and cost, is considered. The constructed simulation model reflects the development, as well as operation effects of the development task, accurately under different resource allocation schemes.

In this study, according to the characteristics of the optimization of the resource allocation problem of an equipment development task, the MOPSO algorithm is selected to optimize. The simulated task under different resource allocation schemes is embedded into the MOPSO algorithm. A multi-objective optimization algorithm for resource allocation of equipment development tasks based on MOPSO is constructed by taking the shortest duration and minimum cost of simulation output as the objective function. Compared with the multi-objective optimization algorithm based on NSGA-II, NSDE and SPEA-II for the resource allocation of the equipment development tasks, the development task cost obtained by the optimization method of this paper is less, which indicates that the optimization effect of the proposed method is better.

The case study demonstrates that the proposed method can optimize the resource allocation according to the specific execution process of the equipment development task, and the optimization effect is obvious. By rationally allocating resources to each sub-task, the degree of resource conflict among the sub-tasks during the execution of development tasks can be greatly reduced, the duration can be shortened, and the cost can be reduced. As the quantity of resources allocated to each sub-task is reduced, the cost of the development task can be reduced, but the development task duration is increased, and a reasonable decision plan needs to be selected according to the duration and cost requirements. Using this method, the resource allocation scheme for an equipment development task can be further improved to a great extent, and the decision makers can provide a scientific basis for the formulation of a resource allocation scheme, thus providing a strong guarantee for the successful completion of the equipment development task.

This paper establishes mathematical models for the relationships between the quantity of resource allocation and duration and cost of a sub-task. A simulation model that can more accurately reflect the real operation of a development task under different resource allocation schemes is constructed. According to the characteristics of the de- 
velopment task resource allocation problem, a resource allocation optimization algorithm based on MOPSO is constructed. The comparison of resource allocation optimization based on different algorithms shows that the optimization effect of the method of this paper is the best. However, in this study, the execution process of the development task is considered in the resource allocation process. However, it is assumed that the quantity of resources allocated to each sub-task during the execution of the development task is fixed. Further research is required to determine a method of allocating resources dynamically to each sub-task according to the execution process of the development task, when the quantity of resources allocated to each sub-task can be adjusted. That is to say, due to many uncertain factors in the development task, its operating parameters and execution process are difficult to predict accurately. It is necessary to dynamically adjust the quantity of resources allocated to each sub-task according to actual needs, and allocate different resources to each sub-task at different stages of execution.

\section{References}

[1] NELSON R G, AZARON A, AREF S. The use of a GERT based method to model concurrent product development processes. European Journal of Operational Research, 2016, 250(2): $566-578$.

[2] DAI W X, WU W W, YU B, et al. Success probability orientated optimization model for resource allocation of the technological innovation multi-project system. Journal of Systems Engineering and Electronics, 2016, 27(6): 1227-1237.

[3] HARTMANN S, BRISKORN D. A survey of variants and extensions of the resource-constrained project scheduling problem. European Journal of Operational Research, 2010, 207(1): $1-14$.

[4] EDEM O P A. Priority rules in project scheduling: a case for random activity selection. Production Planning \& Control, 2000, 11(2): $165-170$.

[5] BROWNING T R, YASSINE A A. Resource-constrained multi-project scheduling: priority rule performance revisited. International Journal of Production Economics, 2010, 126(2): $212-228$.

[6] LI Y C, COIT D. Priority rules-based algorithmic design on two-sided assembly line balancing. Production Engineering, 2018, 12(1): 95-108.

[7] WANG X M, CHEN Q X, MAO N, et al. Proactive approach for stochastic RCMPSP based on multi-priority rule combinations. International Journal of Production Research, 2015, 53(4): $1098-1110$.

[8] CHEN Z, DEMEULEMEESTER E, BAI S J, et al. Efficient priority rules for the stochastic resource-constrained project scheduling problem. European Journal of Operational Research, 2018, 270(3): 957-967.

[9] DIXIT V, VERMA P, RAJ P, et al. Resource and time criticality based block spatial scheduling in a shipyard under uncertainty. International Journal of Production Research, 2018, 56(22): $6993-7007$.

[10] ÖNER-KöZEN M, MINNER S. Impact of priority sequencing decisions on on-time probability and expected tardiness of orders in make-to-order production systems with external due-dates. European Journal of Operational Research, 2017, 263(2): $524-539$.

[11] CHAND S, HUYNH Q, SINGH H, et al. On the use of genetic programming to evolve priority rules for resource constrained project scheduling problems. Information Sciences, 2018, 432: $146-163$.

[12] LI H, ZHANG L, JIAO Y C. Discrete differential evolution algorithm for integer linear bilevel programming problems. Journal of Systems Engineering and Electronics, 2016, 27(4): 912-919.

[13] WANG L, HU H L, AI X Y, et al. Effective electricity energy consumption forecasting using echo state network improved by differential evolution algorithm. Energy, 2018, 153: $801-$ 815.

[14] ZHU A J, XU C P, LI Z, et al. Hybridizing grey wolf optimization with differential evolution for global optimization and test scheduling for 3D stacked SoC. Journal of Systems Engineering and Electronics, 2015, 26(2): $317-328$.

[15] WANG L, LV S X, ZENG Y R, et al. Effective sparse adaboost method with ESN and FOA for industrial electricity consumption forecasting in China. Energy, 2018, 155: 1013-1031.

[16] QI J J, GUO B, LEI H T, et al. Solving resource availability cost problem in project scheduling by pseudo particle swarm optimization. Journal of Systems Engineering and Electronics, 2014, 25(1): 69-76.

[17] RAUNAK M S, OSTERWEIL L J. Resource management for complex, dynamic environments. IEEE Trans. on Software Engineering, 2013, 39(3): $384-402$.

[18] XIONG J, LEUS R, YANG Z Y, et al. Evolutionary multiobjective resource allocation and scheduling in the Chinese navigation satellite system project. European Journal of Operational Research, 2016, 251(2): 662-675.

[19] LIN J T, CHIU C C. A hybrid particle swarm optimization with local search for stochastic resource allocation problem. Journal of Intelligent Manufacturing, 2018, 29(3): 481-495.

[20] YI Y, LI X X, GU C Q. Hybrid particle swarm optimization for multiobjective resource allocation. Journal of Systems Engineering and Electronics, 2008, 19(5): 959-964.

[21] BEŞIKCI U, BILGE Ü, ULUSOY G. Multi-mode resource constrained multi-project scheduling and resource portfolio problem. European Journal of Operational Research, 2015, 240(1): $22-31$.

[22] LIU C C, XIANG X, ZHANG C R, et al. A column generation based distributed scheduling algorithm for multi-mode resource constrained project scheduling problem. Computers \& Industrial Engineering, 2018, 125: 258-278.

[23] KANGASPUNTA J, SALO A. Expert judgments in the costeffectiveness analysis of resource allocations: a case study in military planning. OR Spectrum, 2014, 36(1): $161-185$.

[24] GUO S S, DU B G, PENG Z, et al. Manufacturing resource combinatorial optimization for large complex equipment in group manufacturing: a cluster-based genetic algorithm. Mechatronics, 2015, 31: $101-115$.

[25] CERTA A, ENEA M, GALANTE G, et al. Multi-objective human resources allocation in $R \& D$ projects planning. International Journal of Production Research, 2009, 47(13): $3503-$ 3523.

[26] YAGHOUBI S, NOORI S, AZARONBD A. Resource allocation in dynamic PERT networks with finite capacity. European Journal of Operational Research, 2011, 215(3): 670 - 678.

[27] TAO S, DONG Z S. Multi-mode resource-constrained project scheduling problem with alternative project structures. Computers \& Industrial Engineering, 2018, 125: 333-347.

[28] LASLO Z, GOLDBERG A I. Resource allocation under uncertainty in a multi-project matrix environment: is organizational 
conflict inevitable? International Journal of Project Management, 2008, 26(8): $773-788$.

[29] WANG Y T, HE Z W, KERKHOVE L P, et al. On the performance of priority rules for the stochastic resource constrained multi-project scheduling problem. Computers \& Industrial Engineering, 2017, 114: 223-234.

[30] LEUS R, HERROELEN W. Stability and resource allocation in project planning. IIE Transactions, 2004, 36(7): 667-682.

[31] LIU X Y, LU Z Q. Modeling of and algorithm for resourceconstrained project scheduling problem with resource allocation dependent processing time. Journal of Shanghai Jiaotong University, 2017, 51(1): 82 - 89. (in Chinese)

[32] CHAKRABORTTY R K, SARKER R A, ESSAM D L. Resource constrained project scheduling with uncertain activity durations. Computers \& Industrial Engineering, 2017, 112: $537-550$.

[33] BRUNI M E, PUGLIESE L D P, BERALDI P, et al. An adjustable robust optimization model for the resourceconstrained project scheduling problem with uncertain activity durations. Omega, 2017, 71(C): 66-84.

[34] ZHANG X L, TAN Y J, YANG Z W. Simulation modeling of high-end equipment development task influenced by multiple uncertainty factors. Systems Engineering and Electronics, 2018, 40(6): 1265 - 1273. (in Chinese)

[35] COELlO C A C, PULIDO G T, LECHUGA M S. Handling multiple objectives with particle swarm optimization. IEEE Trans. on Evolutionary Computation, 2004, 8(3): 256-279.

[36] BRITTO A, POZO A. Using reference points to update the archive of MOPSO algorithms in many-objective optimization. Neurocomputing, 2014, 127(3): 78-87.

[37] WANG Y B, ZHAO J M, CHENG Z H, et al. Optimization for spare parts allocation in multi-echelon support system based on improved MOPSO. Systems Engineering and Electronics, 2015, 37(7): 1581 - 1586. (in Chinese)

[38] BROWNING T R, EPPINGER S D. Modeling impacts of process architecture on cost and schedule risk in product development. IEEE Trans. on Engineering Management, 2002, 49(4): $428-442$.

[39] CHO S H, EPPINGER S D. A simulation-based process model for managing complex design projects. IEEE Trans. on Engineering Management, 2005, 52(3): 316-328.

[40] CLERC M. The swarm and the queen: towards a deterministic and adaptive particle swarm optimization. Proc. of the IEEE Congress on Evolutionary Computation, 1999: 1951-1957.

[41] FIGUEIREDO E M N, LUDERMIR T B, BASTOS-FILHO C J A. Many objective particle swarm optimization. Information Sciences, 2016, 374: 115-134.

\section{Biographies}

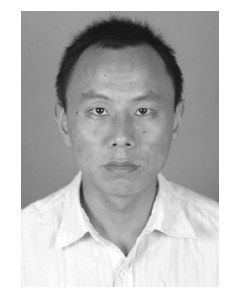

ZHANG Xilin was born in 1984. He received his B.S. degree in logistics engineering from Shandong University in 2007 and M.S. degree in logistics engineering from Jilin University in 2009. He is currently a Ph.D. candidate in College of Systems Engineering, National University of Defense Technology (NUDT). His main research interests include complex system engineering management.

E-mail: zhangxilin16@nudt.edu.cn

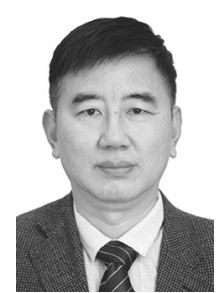

TAN Yuejin was born in 1958. He is currently a professor and $\mathrm{Ph} . \mathrm{D}$. student supervisor in College of Systems Engineering, NUDT. His main research interests include complex system engineering management, and armament system-of-systems technology.

E-mail: yjtan@nudt.edu.cn

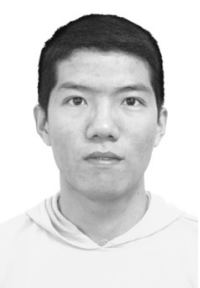

YANG Zhiwei was born in 1988. He received his Ph.D. degree in computer science from Leiden University in 2016. He is currently a lecturer in College of Systems Engineering, NUDT. His main research interests include systems engineering, systems optimization and systems simulation.

E-mail: zhwyang88@hotmail.com 